\title{
Factors influencing maternal microchimerism throughout infancy and its impact on infant
}

\section{T cell immunity}

Christina Balle ${ }^{1}$, Agano Kiravu ${ }^{1}$, Angela Hoffmann ${ }^{1}$, Anna-Ursula Happel ${ }^{1}$, Sami B. Kanaan², J Lee Nelson², Clive M Gray ${ }^{1}$, Heather B Jaspan ${ }^{1,3,4,5}$, Whitney E Harrington ${ }^{3,4 \dagger}$

${ }^{1}$ Division of Immunology, Department of Pathology, Institute of Infectious Disease and Molecular Medicine, University of Cape Town, South Africa, ${ }^{2}$ Clinical Research Division, Fred Hutchinson Cancer Research Center, Seattle, WA, US; ${ }^{3}$ Seattle Children's Research Institute, Seattle, WA, USA; ${ }^{4}$ Department of Pediatrics, University of Washington, Seattle, WA, USA; ${ }^{5}$ Department of Global Health, University of Washington, Seattle, WA, USA

${ }^{+}$Corresponding author

\section{Author contact:}

Whitney Harrington, MD PhD

307 Westlake Ave N

Seattle, WA 98122

Phone: 206 884-1084

Email: whitney.harrington@seattlechildrens.org

\section{Conflict of interest}

SBK and JLN are co-founders of Chimerocyte, Inc. that develops highly-sensitive chimerism analysis technologies. Chimerocyte, Inc. had no role in funding this research project. The remaining authors have declared that no conflict of interest exits. 


\begin{abstract}
Determinants of the acqusition and maintenance of maternal microchimerism (MMc) during infancy and the impact of MMc on infant immune responses are unknown. We examined factors which influence MMc detection and level across infancy and the effect of MMc on T cell responses to BCG vaccination in a cohort of HIV exposed, uninfected and HIV unexposed infants in South Africa. MMc was measured in whole blood from 58 infants using a panel of quantitative PCR assays at day one and 7, 15, and 36 weeks of life. Infants received BCG at birth, and select whole blood samples from infancy were stimulated in vitro with BCG and assessed for polyfunctional CD4+ T cell responses. MMc was present in most infants across infancy at levels ranging from 1-1,193/100,000 genomic equivalents and was positively impacted by absence of maternal HIV, exclusive breastfeeding, and female sex, emphasizing that both maternal and infant factors may shape the maternal graft. Initiation of maternal antiretroviral therapy prior to pregnancy was associated with partial restoration of MMc in HIV exposed, uninfected infants. Birth MMc was associated with an improved polyfunctional CD4+ T cell response to BCG, suggesting that MMc may functionally impact infant immunity.
\end{abstract}




\section{Introduction}

During pregnancy maternal cells are transferred to the fetus and persist in small quantities postnatally, a phenomenon known as maternal microchimerism (MMc). The impact of these maternal cells on the development of the fetal and infant immune system is unknown. MMc is acquired as early as the second trimester (1-3), is found in immune and nonimmune cells throughout the body (4-6), and is maintained into adulthood $(4,7)$. However, there are limited data on factors that determine the quantity of cells a fetus acquires or those that impact the level of MMc across infancy. In a Tanzanian cohort, we recently demonstrated that placental malaria, particularly inflammatory placental malaria, was associated with increased detection and level of cord blood MMc (8). In addition, mode of feeding may shape the maternal graft via at least two potential mechanisms. First, breastfeeding has been associated with increased tolerance of noninherited maternal antigens (NIMA) and the maintenance of MMc in offspring in animal studies (9-12). Second, postnatal transfer of maternal cells to the offspring via breastfeeding has been demonstrated in rodents (13-16) and non-human primates (17), suggesting that the maternal graft in the infant may be a composite of cells acquired transplacentally and via the breastmilk. Maternal HIV, even in the absence of vertical transmission, is associated with placental inflammation, including increased expression of inflammatory cell adhesion molecules, CD8 T cell infiltration (18), chorioamnionitis, and deciduitis (19). Three prior studies have investigated the intersection of MMc and HIV, specifically the risk of vertical transmission, with the most experimentally rigorous identifying an association (20-22). Yet, no studies have looked at the differences in MMc acquisition between infants who are HIV-exposed, uninfected (iHEU) and those who are HIV-unexposed (iHU). For reasons that are still unknown, iHEUs experience increased morbidity and mortality compared to their unexposed counterparts (23). We have previously shown that iHEUs have altered T cell functionality to Bacillus Calmette-Guérin (BCG) and superantigen, including lower interferon (IFN)- $\mathrm{\gamma}$ and polyfunctional cytokine (IFN- $\mathrm{\gamma}$, interleukin (IL)-2 and IL-17) responses (24), suggesting that altered immunity may contribute to their increased morbidity. 
Maternal cells may shape fetal and infant immunity via at least two mechanisms. First, prior work has demonstrated an enrichment of maternal cells in antigen-experienced T cells from cord blood (25), suggesting that the offspring may acquire maternal antigen-specific T cells that could have direct effect when they encounter their cognate antigen in the infant. Second, the acquisition of MMc is associated with the development of NIMA-specific regulatory T cells (Tregs), which suppress effector T cell responses to maternal alloantigens (12). Encounter of neoantigen in the presence of such Tregs may induce cross-tolerance as has been described in transplant biology $(9,26,27)$. Supporting the potential for MMc to impact infant outcomes, we have previously found that detection of cord blood MMc in Tanzanian infants was associated with decreased risk of symptomatic malaria (8), and whole blood MMc detection in American children was found to be protective from the later development of asthma (28). The impact of maternal HIV on MMc and subsequent immune responses in the infant is unknown.

Here, we investigate factors associated with acquisition and maintenance of MMc in South African infants including the potential impact of maternal HIV and mode of feeding. We further assess the impact of MMc on infant $\mathrm{T}$ cell responses directed against BCG, a common neonatal antigen exposure. 


\section{Results}

\section{Cohort characteristics}

HLA and non-HLA typing was performed for 91 mother-infant pairs. Of these, 58 mothers had a unique maternal polymorphism which could be targeted to detect MMc in the background of the offspring. The mean maternal age at delivery was 27 years (standard deviation (sd) 4) (Table 1). This was the first pregnancy for $20.7 \%$ of the mothers (12/58) and the first delivery for $34.5 \%$ (20/58). Estimated gestational age (EGA) at delivery ranged from 36 to 41 weeks (mean 39). Infant birth weight ranged from 2,530 to 4,130 grams (mean 3,207). Just under half of the infants were female $(26 / 58,44.8 \%)$. Nearly two-thirds of the mothers were living with HIV $(37 / 58,63.8 \%)$, of whom just over half $(20 / 37,54.1 \%)$ had received antiretroviral therapy (ART) prior to conception (early ART) while the remaining mothers initiated ART during pregnancy (late ART) at a mean gestational age of 24 weeks (sd 9.6).

Maternal microchimerism at birth is modified by HIV exposure and infant sex

The mean genomic equivalents ( $\mathbf{g E q}$ ) analysed in whole blood samples collected at day one of life (henceforth referred to as birth) was 148,187 (sd 52,867) per sample. At birth, MMc was detectable in 23/58 (39.7\%) infants with a mean level of 1.5/100,000 gEq (Figure 1A-B). In order to identity factors associated with the detection and quantity of maternal cells, we evaluated the potential role of maternal HIV status, maternal age, gravidity, EGA at delivery, and infant sex. The proportion of iHU and iHEU with detectable MMc at birth was similar (iHU: 9/21, 42.9\%, iHEU: 14/37, 37.8\%, unadjusted odds ratio (OR), 0.81 [95\% Cl 0.27-2.44], P=0.713) (Figure 2A, Table 2), and there was a non-statistically significant trend toward lower levels of MMc in iHEU compared to iHU (iHU: mean 6.37/100,000 gEq, iHEU: mean 0.62/100,000 gEq, unadjusted detection rate ratio (DRR), 0.45 [95\% Cl 0.11-1.87], $\mathrm{P}=0.274$ ) (Figure 2B, Table 2). Infants born to mothers with one or more previous pregnancy had similar detection of MMc but a nonsignificantly lower level of MMc at birth of life compared to infants born to women for whom this was their first pregnancy (secondigravidae: unadjusted OR 0.61 [95\% Cl 0.12-3.43], $\mathrm{P}=0.613$; multigravidae: unadjusted OR 0.72 [95\% Cl 0.07-1.12], $\mathrm{P}=0.073$ ) (Table 2). Female infants had 
a non-significantly increased detection of MMc at birth (unadjusted OR 1.65 [95\% $\mathrm{Cl}$ 0.57-4.88], $\mathrm{P}=0.355)$ (Figure 2E, Table 2), but significantly higher levels of MMc than their male counterparts (unadjusted DRR 3.77 [95\% $\mathrm{Cl} 1.14-12.1], \mathrm{P}=0.030$ ) (Figure 2F). Maternal age and EGA at delivery were not associated with MMc at birth. In an adjusted regression model including maternal HIV status, gravidity, and infant sex, the association between HIV exposure and level of $\mathrm{MMc}$ at birth became stronger (adjusted DRR 0.39 [95\% $\mathrm{Cl} 0.14-1.07], \mathrm{P}=0.066$ ) and the impact of infant sex remained significant (adjusted DRR 3.42 [95\% Cl 1.25-9.29], $P=0.016$ ), while the impact of gravidity was attenuated (Table 2 ).

Maternal microchimerism across infancy is dynamic and modified by HIV exposure, mode of feeding, and infant sex

In addition to the birth time point, we also evaluated MMc at week 7, 15 and 36 of life. Across infancy, MMc was detectable at any time point in 44/58 (75.9\%) infants (mean level 16.2/100,000 $\mathrm{gEq}$ ), with a range of detection of 1-3 timepoints (Figure 1A-B). Within individuals, the level of MMc detected across infancy was dynamic (Figure 1C). Levels of MMc higher than typically detected $(>10 / 100,000 \mathrm{gEq})$ were identified at at least one time point in 12/58 (20.7\%) infants, whereas very high MMc (>100/100,000 gEq) was identified at week 7 in one infant $(318 / 100,000$ $\mathrm{gEq}$ ), at week 15 in three infants $(121,729$, and 1193 per 100,000 gEq), and at week 36 in another two infants (338 and 488 per 100,000 gEq) (Figure 1C). Across the group, MMc level increased between birth and 15 weeks, then fell at 36 weeks (Week 7: mean 8/100,000 gEq, week 15: mean 40/100,000 gEq, week 36: mean 17 per 100,000 gEq) (Figure 1A-B).

We assessed whether the covariates associated with $\mathrm{MMc}$ at birth as well as feeding modality and the age of the infant would predict detection and level of MMc across infancy. HIV exposure was associated with a non-statistically significant lower detection (adjusted OR: 0.79 [95\% $\mathrm{Cl}$ 0.38-1.65], $\mathrm{P}=0.530$ ) (Figure 2A) and level of MMc (adjusted DRR: 0.70 [95\% Cl: 0.26-1.92], $\mathrm{p}=0.494$ ) (Figure 2B) (Table 3). Consistent with the birth analysis, female sex was associated with similar detection but higher level of MMc across infancy in both univariate and adjusted models (adjusted DRR 4.53 [95\% Cl 1.46-14.0], $\mathrm{P}=0.009$ ) (Figure 2E-F, Table 3). Formula 
feeding (FF) and mixed feeding (MIF) were associated with lower levels of MMc compared to exclusive breastfeeding (EBF) in both univariate and adjusted models (FF: adjusted DRR 0.36 [95\% Cl 0.12-1.11], $\mathrm{P}=0.074$, MIF: adjusted DRR 0.03 [95\% Cl 0.00-0.23], $\mathrm{P}<0.001$ ) (Figure 2H, Table 3). Infant age was not associated with detection of MMc, but older age was significantly associated with higher level of MMc (adjusted DRR per week: 1.14 [95\% Cl 1.07-1.21], $\mathrm{P}<0.001$ ) (Figure 1B) (Table 3). There was a significant interaction between infant age and maternal HIV status ( $P=0.009)$ with MMc levels increasing faster in iHEU (adjusted DRR per week: 1.16 [95\% $\mathrm{Cl} 1.09-1.24], \mathrm{P}<0.001$ ) versus the iHU group (adjusted DRR per week: 1.08 [95\% Cl 1.02-1.15], $\mathrm{P}=0.005)$, such that by week 15 levels were similar in the two groups.

\section{Initiation of ART prior to conception normalizes maternal microchimerism in iHEU}

We next assessed whether the timing of ART initiation (pre-conception or during gestation) could alter the effect of maternal HIV on MMc. We grouped the infants based on maternal HIV status and timing of ART initiation and repeated the univariate and adjusted analysis. We observed that while both of the HIV-exposed groups had lower levels of MMc compared to the iHUs at birth, this was only significant for infants born to mothers who initated ART during pregnancy (adjusted DRR 0.21 [95\% Cl 0.07-0.60], $\mathrm{P}=0.004$ ) (Table 4). Similarly, iHEUs whose mothers initiated ART during pregnancy had non-significantly lower detection of MMc (adjusted OR 0.56 [95\% Cl 0.241.28], $P=0.170$ ) (Figure 2C, Table 4) and significantly lower level of MMc (adjusted DRR 0.08 [95\% Cl 0.02-0.29], $\mathrm{P}=0.008$ ) (Figure 2D, Table 4) across infancy as compared to iHUs, while iHEUs born to mothers who were on ARV before conception had similar detection and MMc levels to the iHU infants (Figure 2C-D, Table 4). Consistent with these observations, when the two groups of iHEU were compared to each other, there was no difference in detection of birth microchimerism (adjusted $\mathrm{OR}=1.15, \mathrm{p}=0.847$ ), however there was a trend toward higher level amongst early ART verus late ART iHEU (adjusted $D R R=2.42, p=0.141$ ). Similarly, there was a trend toward increased detection (adjusted $\mathrm{OR}=1.85, \mathrm{p}=0.136$ ) and significantly increased level (adjusted DRR=14.54, $\mathrm{p}<0.001$ ) of infancy MMc amongst early verus late ART iHEU. Collectively, these data indicate that longer duration of maternal ART (from pre-conception) appeared to 
partially restore MMc levels in iHEU.

Maternal microchimerism at birth is associated with polyfunctional CD4 T cell responses to BCG Using previously measured BCG vaccine response data from this cohort (29), we sought to determine whether the detection or level of MMc at time of BCG vaccination (birth) would predict T cell responses during early infancy (week 7, week 15, and week 36). We used the COMPASS polyfunctional score (PFS), a clinically validated tool for assessing polyfunctional T cell function (30) to measure CD4+ T cell responses in 36 infants across 64 time points and adjusted for BCG strain (Danish versus Russian), HIV exposure, and infant age in our analyses. Both detection (adjusted coefficient: $0.218, P=0.001$ ) (Figure 3A) and level of MMc (adjusted coefficient per 10/100,000 genomic equivalents: $0.183, P<0.001$ ) at birth (Figure 3 B) positively associated with the PFS. There was a non-significant decline in PFS by age (adjusted coefficient: -0.006 , $\mathrm{P}=0.178$ ) (Figure $\mathbf{3 A}, \mathbf{C}$ ) and a significant interaction between detection of birth $\mathrm{MMc}$ and age $(P=0.049)$, where the rate of decay was greater in those with detectable MMc at birth compared to those without (no birth MMc: adjusted coefficient per week: $-0.001, P=0.852$; yes birth MMc: adjusted coefficient per week: $-0.016, P=0.006$ ) (Figure 4A). We further assessed whether concurrent detection or level of MMc in the infant blood at week 7, 15 or 36 was associated with polyfunctionality of BCG response at the same time point. Unlike birth MMc, detection (Figure 3C) and level (Figure 3D) of concurrent MMc was not associated with PFS. 


\section{Discussion}

This study is the first to measure and compare MMc acquisiton and maintenance in iHEU and iHU during the first year of life. We observed MMc at least at one time point in the majority of infants in the cohort, with high (>10/100,000 gEq) and very high $(>100 / 100,000 \mathrm{gEq})$ levels observed in some infants at the later time points. At birth, HIV exposure and infant sex were associated with MMc. Across infancy, MMc increased up to a peak at 15 weeks of age and was associated with HIV exposure, infant sex, and mode of feeding. Finally, MMc at birth was associated with increased polyfunctionality to BCG later in life, suggesting that these maternal cells may have functional consequence for infant immune responses.

Interestingly, we observed that iHEUs born to mothers who intiated ART pre-conception had higher levels of MMc than those born to mothers who initiated ART during the current pregnancy, and that in the former, levels were comparable to those in iHUs. Recently, in a study using cord blood mononuclear cells collected from term pregnancies, the highest level of MMc was observed among antigen experienced T cells (25). Similarly in mice, T cells were more likely to undergo trans-epithelial migration despite several maternal cell types being detectable in the stomach of pups (15), suggesting that maternal T cells may disproportionately contribute to MMc. Early ART may thus normalize levels of MMc to those seen in unexposed infants through the restoration of maternal CD4 T cells. As MMc acquisition has been observed as early as the beginning of the second trimester $(1,3)$, initiating $A R V s$ during a later antenatal visit may only impact MMc transferred during the later stages of pregnancy. Further, maternal HIV is associated with immune dysregulation and placental inflammation $(18,19)$, which may alter maternal-fetal tolerance and lead to increased rejection of the maternal graft. The reduced MMc conferred by HIV exposure may contribute to abnormal immune responses and increased infectious morbidity in exposed offspring $(23,31,32)$.

We also found that exclusive breastfeeding was associated with higher levels of MMc across infancy compared to formula and mixed feeding, not previously described in humans. These results could be attributed to postnatal transfer of maternal cells via the breastmilk or due to increased maintenance of gestationally-acquired MMc by breastfeeding-reinforced NIMA 
tolerance. Breastfeeding plays a major role in protection from infection in infants through transfer of antibody and other immunomodulatory components (33-36), and recent data from animal models has also demonstrated that breastmilk-derived maternal cells (14-17) including pathogen-experienced immune cells (13) can be detected in the offspring. Our study raises the possibility that postnatal MMc may be acquired through nursing in humans, which may be more pronounced during the early stages of lactation when breast milk cells are more abundant (37) and the infant's gut permeability highest (38). Alternatively, the increased levels of MMc in breastfed infants could be due to increased NIMA-specific tolerance leading to maintenance of in utero acquired MMc. In rodent cross-fostering experiments, pups with no exposure to maternal breast milk lacked tolerance to NIMA-expressing donor allograft tissue (11), had reduced levels of NIMA-specific Tregs (12, 39), and exhibited loss of pregnancy-related MMc (9). Further, the survival of NIMA-matched tissue allografts was reduced among individuals who were not breast fed (10). We observed that at the population level MMc increased with infant age, peaking at week 15 followed by a decline at week 36 . At this time point only one infant remained exclusively breastfed. The reduction in breastfeeding or the introduction of solid foods or formula could lead to a loss in MMc due to increased intestinal or systemic inflammation leading to rejection of maternal cells or due to the lack of continued transfer of maternal breastmilk cells. Distinguishing between these possibilities is challenging in humans.

There are limited data comparing MMc in female and male offspring. Here, we found that female infants had higher levels of MMc than male infants. This observation is consistent with work in mice which found an enrichment of MMc and higher levels of NIMA-specific $T$ regs in female versus male offspring (39). Furthermore, in a cohort of children aged one to seven years born to asthmatic and non-asthmatic mothers, MMc rates were found to be higher in daughters $(24.3 \%)$ compared to sons (16.9\%), albeit these differences did not reach statistical significance (28). Differences in MMc levels between females and males during early infancy could potentially lead to distinct effects on infant immune development which may explain differences in vaccine reponses and disease susceptibility by offspring sex (40). Future studies evaluating MMc in both 
sexes will be needed to further validate any sex-differences in MMc and elucidate the potential mechanism behind this finding.

In order to understand the functional consequence of these rare maternal cells, we asked whether birth or concurrent MMc was associated with polyfunctionality of BCG immune responses in the infant. We were particularly motivated to assess this outcome due to prior data suggesting that HIV exposed infants may mount less functional immune responses to BCG $(24,41,42)$. We identified a positive correlation between $\mathrm{MMc}$ at birth and the polyfuntionality of the $\mathrm{T}$ cell response to BCG vaccination during early infancy. Polyfunctional T cells may be indicative of the quality of immune responses to vaccines and have been associated with better clinical prognosis during tuberculosis (TB), HIV, and other infections as compared to the absolute magnitude of Tcell responses $(30,43-47)$. Since our recruiting site was in Khayalitsha, South Africa, with one of the highest TB rates globally $(48,49)$, we estimate that approximately $80 \%$ of the pregnant women in our study were infected with TB, with the majority in latent form. We hypothesize that given the prior finding of an enrichment of MMc among antigen experienced T-cells (25), TB-specific T cells may transfer from latently infected mothers to their infants, directly contributing to or shaping the infant response to $B C G$ vaccination. This observation together with the finding of decreased MMc in iHEU suggests that limited MMc may play a role in diminished BCG-specific responses in these infants. In contrast, we found no association between concurrent MMc and BCG response which may reflect differences in the type and specificity of maternal cells acquired via the placenta or via breastmilk or alternatively may reflect the decay of maternal TB-specific T cells with time.

While the level of MMc we identified in most infants at any time points was similar to prior work $(0-10 / 100,000 \mathrm{gEq})(6,50)$, in a proportion of infants we observed very high levels of MMc at select time points, such that up to $1 \%$ of cells appeared to be of maternal origin, many orders of magnitude higher than previously described. To the best of our knowledge, this is the first description of such exceptionally high levels of maternal cells in infants, and raises many essential follow up questions about the origin, phenotype, and function of these cells. Specifically, we hypothesize that these discrete high levels of MMc may represent maternal cell proliferation in 
response to an exogenous stimulus (e.g. infection or vaccination in the infant), which should be evaluated in future studies.

Our study has a number of limitations. First, our sample size was predominantly limited to maternal-infant pairs where there was a non-shared non-inherited maternal HLA Class II allele. This limited our ability to study MMc in maternal-infant pairs with a high degree of HLA Class II concordance. Since HLA plays a critical role in regulating immune tolerance, MMc could be influenced by the HLA relationship between mother and fetus as has been shown in animal models (51). In addition BCG response data was only available for a subset of the cohort, however, there were no appreciable differences between those infants with and without data available.

To our knowledge, this is the first study to assess maternal microchimerism in infants across the first year of life and relate their levels to vaccine response. Our findings highlight the as yet largely unexplored impact of this cross-generation graft of maternal cells on infant immunity. In addition, our findings provide insight into an additional potential mechanism that may contribute to altered immune responses in HIV exposed but uninfected infants and the importance of maternal immune restoration to impact infant outcome. Future work should investigate the cellular phenotype and antigen-specificity of these inherited maternal cells. 


\section{Materials and Methods}

\section{Study design}

Data and samples were utilized from the Innate Factors Associated with Nursing Transmission (InFANT), an ongoing prospective birth cohort conducted in Khayelitsha, Cape Town, South Africa funded by the Canadian Institute for Health Research (Co-PIs Jaspan, Gray). Mother-infant pairs were recruited at the Midwifery Obstetric Unit at Site B in Khayelitsha, Cape Town, South Africa from March 2014 to March 2018. Infants were followed from birth, at day 4-7, and at weeks 7, 15, and 36 of life. Voluntary counseling and HIV testing was done at the time of antenatal care registration. Both pregnant women living with HIV (WLHIV) and women not living with HIV were eligible for the study. Pregnant WLWH and their infants were provided with anti-retrovirals (ARV) according to the current country-specific guidelines (52). Exclusive breastfeeding was advised to all mothers from delivery to at least 6 months. Infants born before 36 weeks of gestation and with birth weights lower than $2.4 \mathrm{~kg}$ were ineligible for the study. Further exclusion criteria included pregnancy or delivery complications as previously described (53). All infants who were HIVexposed uninfected were confirmed as HIV negative by PCR at delivery and at later time points according to prevention of vertical transmission guidelines (52). Routine vaccines were given to all infants according to the WHO's Expanded Program on Immunization (EPI). Infants received intradermal Danish BCG strain (1331, Statens Serum Institute, Denmark) from April 2013 to January 2016 and thereafter Russian strain (BCG-I Moscow, Serum Institute of India, India). Both strains were given at $2 \times 10^{\wedge} 5 \mathrm{CFU} /$ dose at birth. Maternal-infant pairs were included in the present study based on available paired maternal and infant samples $(\mathrm{N}=91)$ and informative typing for a maternal-specific allele with a developed assay available $(\mathrm{N}=58)$.

\section{Genomic DNA Extraction}

Whole blood samples were collected at day one (birth) and weeks 7, 15, and 36 of life into Sodium-Heparin tubes. A $200 \mu \mathrm{l}$ aliquot of the whole blood was lysed using FACS lysis buffer and subsequently fixed and stored at $-80^{\circ} \mathrm{C}$ until genomic DNA (gDNA) extraction. The gDNA was extracted from fixed whole blood (WBF) samples from infants and mothers using QiaAMP DNA 
Mini Kits (Qiagen) with slight modifications to the manufaturer's protocol. In brief, WBF samples were thawed and centrifuged at $16,000 \mathrm{rpm}$ for 2 mins to pellet cells. The supernatant was discarded and the pellet re-suspended in $180 \mu \mathrm{L}$ Qiagen Buffer ATL and $20 \mu \mathrm{L}$ Proteinase K followed by incubation at at $56^{\circ} \mathrm{C}$ for one hour. This was followed by addition of $200 \mu \mathrm{L}$ Buffer $\mathrm{AL}$ and $200 \mu \mathrm{L} 100 \%$ ethanol and the entire sample lysate was transferred onto the QIAamp MinElute column. The gDNA was eluted using $200 \mu \mathrm{L}$ molecular grade water which was allowed to incubate on the membrane for 10 mins at room temperature to increase DNA yield.

\section{Identification of Maternal-Specific Polymorphisms and Microchimerism Evaluation}

Genomic DNA from maternal and infant granulocytes collected at week 36 was genotyped at HLA class II loci DRB1, DQA1, and DQB1 by direct sequencing (Scisco Genetics, Seattle, WA). Noninherited, nonshared HLA polymorphisms were identified that could be used to evaluate MMc $(54,55)$. Maternal-infant pairs with non-informative HLA typing were typed at 4 non-HLA loci: GSTT1, ATIII, TG, and TNN, targeting insertion/deletion polymorphisms (25). The maternalspecific polymorphism identified for each maternal-infant pair was selectively amplified from WBF gDNA using a panel of previously developed qPCR assays $(25,54,56)$. The sensitivity of each of the qPCR assays is one target genomic equivalent $(\mathrm{gEq})$ in 60,000 background $\mathrm{gEq}$ per reaction well (57). DNA from each WBF was distributed in 6 to 16 reaction wells, dependent upon sample concentration, with the objective of reaching a total gEq of 120,000 per sample. A calibration curve for the polymorphism-specific assay was included to quantify the amount of MMc for each experiment. Every sample was also quantified by the nonpolymorphic $\beta$-globin gene; a $\beta$-globin calibration curve (obtained from standard human genomic DNA [Promega]) was concurrently evaluated on each plate to quantify the total number of gEq of DNA tested in each reaction. Level of MMc was calculated according to the number of $\mathrm{gEq}$ of microchimeric cells (estimated by the target gene calibration curve) proportional to the number of $\mathrm{gEq}$ of total cells (estimated by the reference gene calibration curve) and was expressed as MMc gEq per $10^{5} \mathrm{gEq}$ tested. 


\section{Whole Blood BCG stimulation Assay}

A 12-hour whole blood assay was used to evaluate vaccine responses as previously described (58). Briefly, $250 \mu \mathrm{L}$ of anticoagulated blood was incubated with $\mathrm{BCG}$, media or PHA at $37^{\circ} \mathrm{C}$ within $1 \mathrm{~h}$ of blood draw. After $5 \mathrm{~h}$, Brefeldin-A (Sigma Aldrich) was added and incubated for an additional $7 \mathrm{~h}$. Thereafter, red blood cells were lysed followed by washing and staining with LIVE/DEAD ${ }^{\circledR}$ fixable Violet stain (ViViD, ThermoFisher). The cells were cryo-preserved in liquid nitrogen $\left(\mathrm{LN}_{2}\right)$.

\section{Cell Staining, Antibodies, and Flow Cytometry}

Staining and flow cytometry were conducted as previously described (29). In brief, batched stored samples were thawed quickly and washed, incubated in BD PermWash and then stained with the antibody cocktail mix. After incubation, cells were washed and the resuspended in PBS for cell acquisition using a Beckton Dickinson LSRII flow cytometer (SORF model). The following monoclonal antibody-fluorochrome conjugates were used: IL-2-R-phycoerythrin (PE), CD8-V500, IFN-y-Alexa Fluor-700, TNF-a-PE-Cy7, Ki67-Fluorescein isothiocyanate (FITC), all from BD, CD27 PE-Cy5, HLA-DR- Allophycocyanin-Cy7 (APC-Cy7), CD3-BV650 (Biolegend), CD4 PECy5.5 (Invitrogen), CD45RA PE-Texas Red-X (Beckman Coulter). A minimum of 50,000 ViViD negative (viable) CD3 events were collected using FACS DIVA v6 software. Post-acquisition compensation and analysis was performed in FlowJo version 9 (FlowJo, LLC). Supplementary Figure 1 shows the gating strategy employed. Measured response to BCG was characterized by the polyfunctionality of the CD3+CD4+ T cell response assessed by analyzing permutations of TNF- $\alpha$, IL-2 and IFN- $y$ expression after stimulation using COMPASS (Combinatorial polyfunctionality analysis of antigen-specific T-cell subsets) (30). Polyfunctionality scores (PFS) which summarize the functional profile of each subject were calculated from posterior probabilities as described (30). 


\section{Statistics}

Statistical analysis was performed using Stata 14 software (StataCorp LP) and R (version 4.0.3) and figures generated in R. Detection of MMc at birth was evaluated by logistic regression with adjustment for the total number of gEq tested for each subject. Level of MMc at birth was evaluated with negative binomial regression, accounting for the number of microchimeric $\mathrm{gEq}$ detected as well as the total number of $\mathrm{gEq}$ assessed in each sample. This approach accounts for the nonnormal distribution of MMc data as well as the large number of zeros (59). The output of this model is a detection rate ratio (DRR), which can be interpreted as " $X$ number of microchimeric gEq in group A for every one microchimeric gEq in group B." Each covariate of interest was first tested individually in univariate models. For the adjusted model, we included HIV exposure a priori as it was a primary covariate of interest and included other covariates if they were associated with either detection or level of $\mathrm{MMc}$ at the $\mathrm{P}<0.1$ level (infant sex and gravidity). Detection of MMc across infancy was assessed using generalized estimating equation (GEE) models clustered by individual, a binomial outcome structure, and an exchangeable correlation matrix. Level of MMc across infancy was assessed using GEE models clustered by individual, a negative binomial outcome structure, and an exchangeable correlation matrix. In addition to the covariates mentioned above (HIV exposure, infant sex, gravidity), mode of feeding and infant age in weeks were considered for inclusion in the adjusted models. To assess differences in the rate of change in MMc across infancy in HIV-exposed and unexposed infants, we generated an interaction term between maternal HIV and infant age for inclusion in the model.

The effect of MMc on the polyfunctionality of BCG vaccine responses (PFS) were assessed using GEE models clustered by individual, a gaussian outcome structure, and an exchangeable correlation matrix. We have previously shown that iHEU infants have altered $\mathrm{T}$ cell functionality to BCG (24) and that the type of BCG strain used for vaccination contributes strongly to the magnitude and polyfunctionality of vaccine response elicited in infants (29). We therefore adjusted for BCG strain, HIV exposure, and infant age in our analyses. To access differences in the rate of change in BCG vaccine responses across infancy in infants with or without MMc 
detected at birth, we generated an interaction term between birth detectable MMc and infant age for inclusion in the model.

\section{Study approval}

The study was approved by the UCT Human Research Ethics Committee (Rec ref 285/2012) and the Institutional Review Board of Seattle Children's Hospital (Protocol \#15690). All mothers in the study were of consenting age and provided written informed consent for their and their infant's participation.

\section{Author contributions}

CB, HBJ, SBK, CMG, JLN, and WEH conceived and designed the experiments. HBJ and CMG designed and recruited the InFANT cohort. $\mathrm{CB}, \mathrm{AK}, \mathrm{AHO}, \mathrm{AHA}$ and $\mathrm{JH}$ processed samples and performed wet lab experiments. $\mathrm{CB}, \mathrm{WH}$ and $\mathrm{AK}$ analyzed the data. $\mathrm{CB}, \mathrm{HBJ}$ and WEH wrote the original draft of the manuscript. All authors reviewed and edited the manuscript.

\section{Acknowledgements}

The study was funded by National Institutes of Health/University of Washington Center for AIDS Research grant Al027757 (WEH), National Institutes of Health/National Institute of Allergy and Infectious Diseases grant K08 Al135072 (WEH), Burroughs Wellcome Fund award number CAMS 1017213 (WEH), Global Health Research Initiative (GHRI) award number THA-118568 (HBJ, CGM), National Institutes of Health grant R01Al120714-01A1 (HJ) and National Institutes of Health grant U01Al131302 (HBJ, CGM). We would like to thank the women and infants from Khayelitsha, Cape Town, South Africa for their participation in the InFANT cohort and recognize the clinic staff at Site B Khayelitsha and the InFANT clinical team for their care of study participants. We also acknowledge the Western Cape Department of Health for access to their facilities. 


\section{References}

1. Lo ES et al. Transfer of nucleated maternal cells into fetal circulation during the second trimester of pregnancy.. Br. J. Haematol. 1998;100(3):605-606.

2. Gammill HS et al. Pregnancy, Microchimerism, and the Maternal Grandmother [Internet]. PLoS One $2011 ; 6(8): \mathrm{e} 24101$.

3. Jonsson AM et al. Maternal microchimerism in human fetal tissues [Internet]. Am. J. Obstet. Gynecol. 2008;198(3):325.e1-325.e6.

4. Loubiere LS et al. Maternal microchimerism in healthy adults in lymphocytes, monocyte/macrophages and NK cells.. Lab. Invest. 2006;86(11):1185-1192.

5. Hall JM et al. Detection of maternal cells in human umbilical cord blood using fluorescence in situ hybridization.. Blood 1995;86(7):2829-2832.

6. Nelson JL. The otherness of self: microchimerism in health and disease.. Trends Immunol. 2012;33(8):421-427.

7. Maloney S et al. Microchimerism of maternal origin persists into adult life.. J. Clin. Invest. 1999;104(1):41-47.

8. Harrington WE et al. Maternal Microchimerism Predicts Increased Infection but Decreased Disease due to Plasmodium falciparum During Early Childhood.. J. Infect. Dis. 2017;215(9):14451451.

9. Dutta P et al. Microchimerism is strongly correlated with tolerance to noninherited maternal antigens in mice.. Blood 2009;114(17):3578-3587.

10. Campbell DAJ et al. Breast feeding and maternal-donor renal allografts. Possibly the original donor-specific transfusion.. Transplantation 1984;37(4):340-344.

11. Andrassy $\mathrm{J}$ et al. Tolerance to noninherited maternal MHC antigens in mice.. J. Immunol. 2003;171(10):5554-5561.

12. Mold JE et al. Maternal alloantigens promote the development of tolerogenic fetal regulatory T cells in utero.. Science 2008;322(5907):1562-1565.

13. Darby MG et al. Pre-conception maternal helminth infection transfers via nursing long-lasting cellular immunity against helminths to offspring.. Sci. Adv. 2019;5(5):eaav3058. 
14. Aydın MŞ et al. Transfer and Integration of Breast Milk Stem Cells to the Brain of Suckling Pups.. Sci. Rep. 2018;8(1):14289.

15. Ma LJ et al. Trans-epithelial immune cell transfer during suckling modulates delayed-type hypersensitivity in recipients as a function of gender.. PLoS One 2008;3(10):e3562.

16. Zhou L et al. Two independent pathways of maternal cell transmission to offspring: through placenta during pregnancy and by breast-feeding after birth.. Immunology 2000;101(4):570-580. 17. Jain $L$ et al. In vivo distribution of human milk leucocytes after ingestion by newborn baboons.. Arch. Dis. Child. 1989;64(7 Spec No):930-933.

18. Baurakiades $E$ et al. Histomorphometric and immunohistochemical analysis of infectious agents, T-cell subpopulations and inflammatory adhesion molecules in placentas from HIVseropositive pregnant women.. Diagn. Pathol. 2011;6:101.

19. Schwartz DA et al. Placental abnormalities associated with human immunodeficiency virus type 1 infection and perinatal transmission in Bangkok, Thailand.. J. Infect. Dis. 2000;182(6):1652-1657. 20. Biggar RJ et al. The role of transplacental microtransfusions of maternal lymphocytes in HIV transmission to newborns.. AIDS 2008;22(17):2251-2256.

21. Lee $\mathrm{T}-\mathrm{H}$ et al. The role of transplacental microtransfusions of maternal lymphocytes in in utero HIV transmission.. J. Acquir. Immune Defic. Syndr. 2010;55(2):143-147.

22. Kwiek JJ et al. Maternal-fetal DNA admixture is associated with intrapartum mother-to-child transmission of HIV-1 in Blantyre, Malawi.. J. Infect. Dis. 2008;197(10):1378-1381.

23. Marinda E et al. Child mortality according to maternal and infant HIV status in Zimbabwe.. Pediatr. Infect. Dis. J. 2007;26(6):519-526.

24. Kidzeru EB et al. In-utero exposure to maternal HIV infection alters T-cell immune responses to vaccination in HIV-uninfected infants.. AIDS 2014;28(10):1421-1430.

25. Kanaan SB et al. Maternal microchimerism is prevalent in cord blood in memory $\mathrm{T}$ cells and other cell subsets, and persists post-transplant.. Oncoimmunology 2017;6(5):e1311436.

26. Bemelman $\mathrm{F}$ et al. Bone marrow transplantation induces either clonal deletion or infectious tolerance depending on the dose.. J. Immunol. 1998;160(6):2645-2648.

27. Qin S et al. “Infectious" transplantation tolerance.. Science 1993;259(5097):974-977. 
28. Thompson EE et al. Maternal microchimerism protects against the development of asthma [Internet]. J. Allergy Clin. Immunol. 2013;132(1):39-44.e4.

29. Kiravu A et al. Bacille Calmette-Guérin Vaccine Strain Modulates the Ontogeny of Both Mycobacterial-Specific and Heterologous T Cell Immunity to Vaccination in Infants.. Front. Immunol. $2019 ; 10: 2307$

30. Lin L et al. COMPASS identifies T-cell subsets correlated with clinical outcomes.. Nat. Biotechnol. 2015;33(6):610-616.

31. Brennan AT et al. A meta-analysis assessing all-cause mortality in HIV-exposed uninfected compared with HIV-unexposed uninfected infants and children.. AIDS 2016;30(15):2351-2360.

32. Evans C, Jones CE, Prendergast AJ. HIV-exposed, uninfected infants: new global challenges in the era of paediatric HIV elimination.. Lancet. Infect. Dis. 2016;16(6):e92-e107.

33. Goldman AS. The immune system of human milk: antimicrobial, antiinflammatory and immunomodulating properties.. Pediatr. Infect. Dis. J. 1993;12(8):664-671.

34. Hanson LA. Breastfeeding provides passive and likely long-lasting active immunity.. Ann. allergy, asthma Immunol. Off. Publ. Am. Coll. Allergy, Asthma, Immunol. 1998;81(6):523-524,537. 35. Kleist SA, Knoop KA. Understanding the Elements of Maternal Protection from Systemic Bacterial Infections during Early Life.. Nutrients 2020;12(4). doi:10.3390/nu12041045 36. Victora CG et al. Breastfeeding in the 21st century: epidemiology, mechanisms, and lifelong effect.. Lancet (London, England) 2016;387(10017):475-490.

37. Goldman AS et al. Immunologic factors in human milk during the first year of lactation.. J. Pediatr. 1982;100(4):563-567.

38. Catassi $\mathrm{C}$ et al. Intestinal permeability changes during the first month: effect of natural versus artificial feeding.. J. Pediatr. Gastroenterol. Nutr. 1995;21(4):383-386.

39. Kinder JM et al. Cross-Generational Reproductive Fitness Enforced by Microchimeric Maternal Cells.. Cell 2015;162(3):505-515.

40. Flanagan KL et al. Sex and Gender Differences in the Outcomes of Vaccination over the Life Course.. Annu. Rev. Cell Dev. Biol. 2017;33:577-599.

41. Hesseling AC et al. Immunogenicity of BCG in HIV-exposed and non-exposed infants following 
routine birth or delayed vaccination.. Int. J. Tuberc. lung Dis. Off. J. Int. Union against Tuberc. Lung Dis. 2015;19(4):454-462.

42. Jones CE et al. The impact of HIV exposure and maternal Mycobacterium tuberculosis infection on infant immune responses to bacille Calmette-Guérin vaccination [Internet]. AIDS 2015;29(2).https://journals.Iww.com/aidsonline/Fulltext/2015/01140/The_impact_of_HIV_exposure_ and_maternal.3.aspx. cited

43. Beveridge NER et al. Immunisation with BCG and recombinant MVA85A induces long-lasting, polyfunctional Mycobacterium tuberculosis-specific CD4+ memory T lymphocyte populations.. Eur. J. Immunol. 2007;37(11):3089-3100.

44. Darrah PA et al. Multifunctional TH1 cells define a correlate of vaccine-mediated protection against Leishmania major.. Nat. Med. 2007;13(7):843-850.

45. Ciuffreda D et al. Polyfunctional HCV-specific T-cell responses are associated with effective control of HCV replication.. Eur. J. Immunol. 2008;38(10):2665-2677.

46. Rodrigue-Gervais IG et al. Dendritic cell inhibition is connected to exhaustion of CD8+ T cell polyfunctionality during chronic hepatitis C virus infection.. J. Immunol. 2010;184(6):3134-3144.

47. Precopio ML et al. Immunization with vaccinia virus induces polyfunctional and phenotypically distinctive CD8(+) T cell responses.. J. Exp. Med. 2007;204(6):1405-1416.

48. WHO. Global tuberculosis report 2020. Geneva: World Health Organization; 2020. Licence: CC BY-NC-SA 3.0 IGO. 2020:

49. Mahtab S, Coetzee D. Influence of HIV and other risk factors on tuberculosis.. S. Afr. Med. J. 2017;107(5):428-434.

50. Gammill HS, Harrington WE. Microchimerism: Defining and redefining the prepregnancy context - A review.. Placenta 2017;60:130-133.

51. Kaplan J, Land S. Influence of maternal-fetal histocompatibility and MHC zygosity on maternal microchimerism.. J. Immunol. 2005;174(11):7123-7128.

52. National Department of Health South Africa 2015. National Consolidated Guidelines2015;(November):1-78.

53. Tchakoute CT et al. Breastfeeding mitigates the effects of maternal HIV on infant infectious 
morbidity in the Option B+ era.. AIDS 2018;32(16):2383-2391.

54. Lambert NC et al. Quantification of maternal microchimerism by HLA-specific real-time polymerase chain reaction: studies of healthy women and women with scleroderma.. Arthritis Rheum. 2004;50(3):906-914.

55. Loubière LS et al. Maternal microchimerism in healthy adults in lymphocytes, monocyte/macrophages and NK cells.. Lab. Invest. 2006;86(11):1185-1192.

56. Gammill HS et al. Effect of parity on fetal and maternal microchimerism: interaction of grafts within a host?. Blood 2010;116(15):2706-2712.

57. Kanaan SB et al. Immunogenicity of a rheumatoid arthritis protective sequence when acquired through microchimerism.. Proc. Natl. Acad. Sci. U. S. A. 2019;116(39):19600-19608.

58. Hanekom WA et al. Novel application of a whole blood intracellular cytokine detection assay to quantitate specific T-cell frequency in field studies.. J. Immunol. Methods 2004;291(1-2):185-195. 59. Guthrie KA et al. Statistical Methods for Unusual Count Data: Examples From Studies of Microchimerism.. Am. J. Epidemiol. 2016;184(10):779-786. 


\section{Figures}
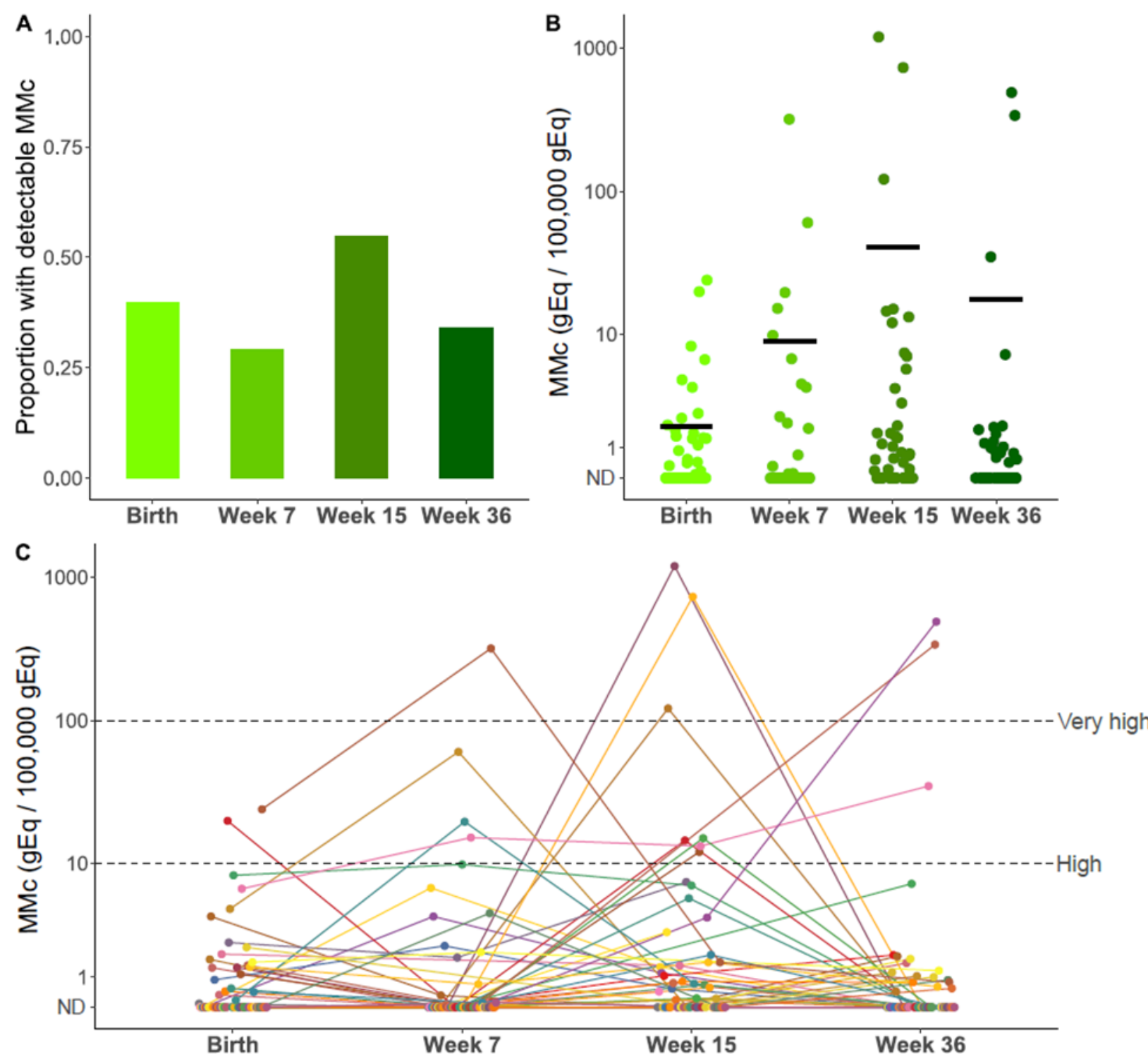

Figure 1: Maternal microchimerism is dynamic and persist throughout infancy. Maternal microchimiersm (MMc) expressed as microchimeric equivalents per infant genomic equivalents ( $\mathrm{gEq}$ ) assessed. Not detected (ND). (A) Propotion of infants with detectable MMc at birth $(\mathrm{N}=58)$, week $7(\mathrm{~N}=55)$, week $15(\mathrm{~N}=53)$, and week $36(\mathrm{~N}=53)$ of life. (B) Quantitative levels of MMc across infancy. Horizontal black lines indicate mean value. (C) Within infant MMc kinetics. The dotted lines highlight MMc levels above 10/100,000 gEq (High) and above 100/100,000 gEq (Very high). 

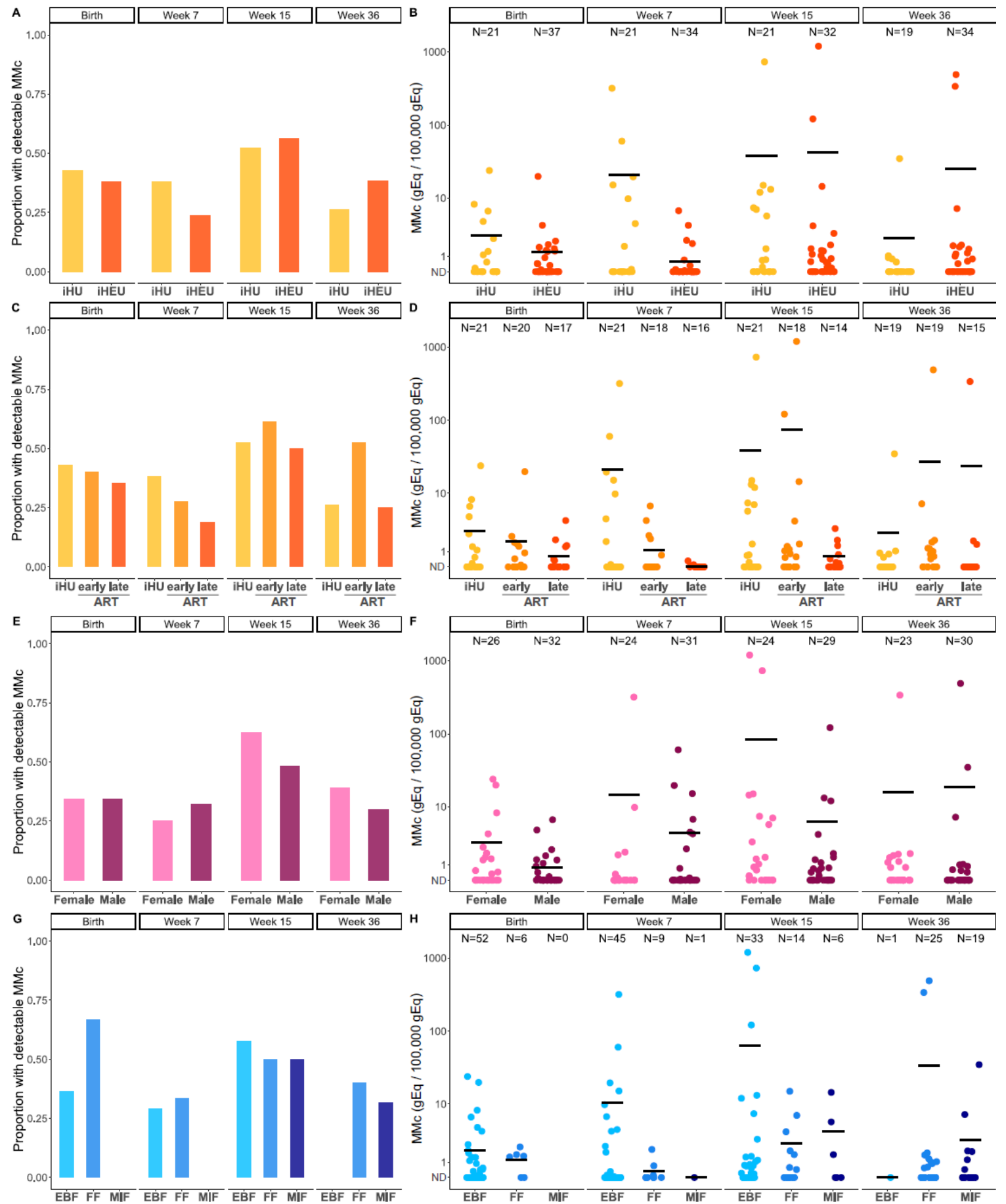

Figure 2. Maternal microchimerism across infancy is modified by HIV exposure, duration of maternal antiretroviral therapy (ART), infant sex, and mode of feeding. Maternal microchimerism (MMc) expressed as microchimeric equivalents per infant genomic equivalents assessed. Not detected (ND), HIV-unexposed infants (iHU), HIV-exposed uninfected infants (iHEU), HIV-exposed uninfected infants whose mothers were on antiretroviral therapy (ART) preconception (early ART), HIV-exposed uninfected infants whos mothers initiated ART during pregnancy (late ART), exclusively breastfed (EBF), formula fed (FF), mixed infant feeding (MIF). Detection of MMc in infants at day one, week 7, week 15 and week 36 of life by HIV exposure (A), HIV exposure and duration of ART (C), infant sex (E), and mode of feeding (G). Level of MMc across infancy by HIV exposure (B), HIV exposure and duration of ART (D), infant sex (F), and mode of feeding $(\mathrm{H})$. Horizontal black lines indicate mean value. 

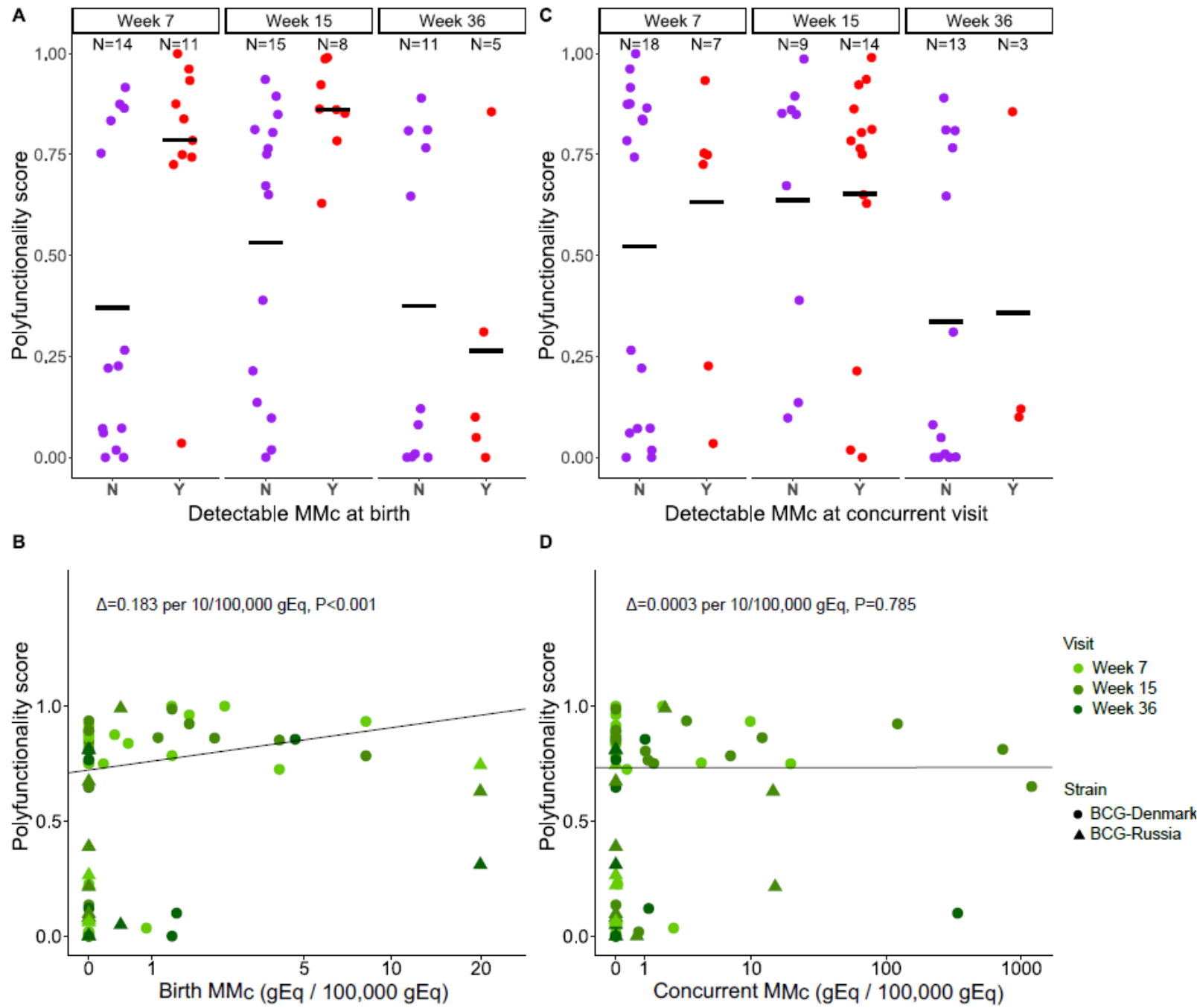

Figure 3. Maternal microchimerism at birth is positively associated with $\mathrm{T}$ cell responses to BCG vaccination. (A) Polyfunctionality score (PFS) at week 7, week 15 and week 36 of life by detection of maternal microchimiersm (MMc) at birth ( $\mathrm{N}=$ No (purple), $\mathrm{Y}=\mathrm{Yes}(\mathrm{red})$ ). Correlation of PFS at week 7 (light green), week 15 (green) and week 36 (dark green) of life with level of MMc per 100,000 genomic equivalents $(\mathrm{gEq})$ at birth. Delta represent the adjusted effect size per 10/100,000 gEq. (C) PFS at week 7, week 15 and week 36 of life by detection of MMc at the concurrent time point $(\mathrm{Y}=\mathrm{Yes}$ (red), $\mathrm{N}=\mathrm{No}$ (purple)). (D) Correlation of PFS at week 7 (light green), week 15 (green) and week 36 (dark green) of life by level of MMc per 100,000 genomic equivalents $(\mathrm{gEq})$ at the concurrent time point. Delta represent the adjusted effect size per 10/100,000 gEq. Horizontal black lines indicate mean value. 


\section{Tables}

Table 1. Cohort characteristics

\begin{tabular}{|l|c|}
\hline & $\begin{array}{c}\text { Total } \\
(\mathbf{n}=58)\end{array}$ \\
\hline Maternal age years, mean (sd) & $27(4)$ \\
\hline Gravidity, $\mathbf{n}(\%)$ & $12(20.7)$ \\
Primigravidae & $25(43.1)$ \\
Secondigravidae & $21(36.2)$ \\
Multigravidae & $26(44.8)$ \\
\hline Infant sex, female, $\mathbf{n}(\%)$ & $3207(416)$ \\
\hline Birth weight, grams, mean (sd) & $39(1.35)$ \\
\hline Gestational age at delivery, weeks, mean (sd) & $58(100)$ \\
\hline Delivery method, vaginal, $\mathbf{n}(\%)$ & $37(63.8)$ \\
\hline HIV exposed, $\mathbf{n}(\%)$ & \\
\hline Maternal antiretroviral therapy & $20 / 37(54.1)$ \\
Prior to pregnancy, $\mathrm{n}(\%)$ & $17 / 37(45.9)$ \\
Initiated during pregnancy, $\mathrm{n}(\%)$ & $24(9.6)$ \\
$\quad-\quad$ Gestational age at initiation, weeks, mean & \\
\hline (sd) & $39(67.2)$ \\
BCG strain used for vaccination & $19(32.8)$ \\
\hline BCG-Russia, $n$ (\%) &
\end{tabular}


Table 2. Association between covariates and detection and level of maternal microchimerism at birth.

\begin{tabular}{|c|c|c|c|c|}
\hline & \multicolumn{4}{|c|}{ Detection of MMc at birth } \\
\hline & OR $(95 \% \mathrm{Cl})$ & $P$ value & Adj. OR $(95 \% \mathrm{Cl})$ & $P$ value \\
\hline Maternal HIV & $0.81(0.27-2.46)$ & 0.713 & $0.80(0.25-2.54)$ & 0.698 \\
\hline Maternal age & $0.99(0.89-1.09)$ & 0.778 & - & - \\
\hline $\begin{array}{l}\text { Gravidity } \\
\text { Primigravidae } \\
\text { Secondigravidae } \\
\text { Multigravidae }\end{array}$ & $\begin{array}{c}\text { REF } \\
0.95(0.23-4.06) \\
0.88(0.20-3.95)\end{array}$ & $\begin{array}{l}- \\
0.940 \\
0.861\end{array}$ & $\begin{array}{c}\text { REF } \\
1.09(0.25-4.92) \\
1.09(0.23-5.36)\end{array}$ & $\begin{array}{l}- \\
0.910 \\
0.916\end{array}$ \\
\hline EGA & $1.09(0.73-1.63)$ & 0.677 & - & - \\
\hline \multirow[t]{3}{*}{ Infant sex, female } & $1.65(0.57-4.88)$ & 0.355 & $1.69(0.56-5.22)$ & 0.349 \\
\hline & \multicolumn{4}{|c|}{ Level of MMc at birth } \\
\hline & DRR (95\% Cl) & $P$ value & Adj. DRR (95\% Cl) & $P$ value \\
\hline Maternal HIV & $0.45(0.11-1.87)$ & 0.274 & $0.39(0.14-1.07)$ & 0.066 \\
\hline Maternal age & $1.02(0.82-1.27)$ & 0.857 & - & - \\
\hline $\begin{array}{l}\text { Gravidity } \\
\text { Primigravidae } \\
\text { Secondigravidae } \\
\text { Multigravidae } \\
\end{array}$ & $\begin{array}{c}\text { REF } \\
0.61(0.12-3.43) \\
0.72(0.07-1.12) \\
\end{array}$ & $\begin{array}{l}- \\
0.613 \\
0.073 \\
\end{array}$ & $\begin{array}{c}\text { REF } \\
0.47(0.11-1.99) \\
0.44(0.10-1.89) \\
\end{array}$ & $\begin{array}{l}- \\
0.307 \\
0.267 \\
\end{array}$ \\
\hline EGA & $0.92(0.61-1.38)$ & 0.670 & - & - \\
\hline Infant sex, female & $3.77(1.14-12.1)$ & 0.030 & $3.42(1.25-9.29)$ & 0.016 \\
\hline
\end{tabular}


bioRxiv preprint doi: https://doi.org/10.1101/2021.03.02.432825; this version posted March 2, 2021. The copyright holder for this preprint (which was not certified by peer review) is the author/funder. All rights reserved. No reuse allowed without permission.

Table 3. Association between covariates and detection and level of maternal microchimerism across infancy.

\begin{tabular}{|c|c|c|c|c|}
\hline & \multicolumn{4}{|c|}{ Detection of MMc across infancy } \\
\hline & OR $(95 \% \mathrm{Cl})$ & $\mathbf{P}$ value & Adj. OR (95\% Cl) & $P$ value \\
\hline Maternal HIV & $0.91(0.44-1.86)$ & 0.790 & $0.79(0.38-1.65)$ & 0.530 \\
\hline $\begin{array}{l}\text { Gravidity } \\
\text { Primigravidae } \\
\text { Secondigravidae } \\
\text { Multigravidae }\end{array}$ & $\begin{array}{c}\text { REF } \\
1.46(0.56-3.79) \\
1.81(0.67-4.94)\end{array}$ & $\begin{array}{c}- \\
0.440 \\
0.240\end{array}$ & $\begin{array}{c}\text { REF } \\
1.48(0.52-4.23) \\
2.01(0.69-5.85)\end{array}$ & $\begin{array}{c}- \\
0.460 \\
0.200\end{array}$ \\
\hline Infant sex, female & $1.38(0.71-2.67)$ & 0.350 & $1.58(0.76-3.29)$ & 0.220 \\
\hline $\begin{array}{l}\text { Feeding } \\
\text { Exclusive breastfeeding } \\
\text { Formula feeding } \\
\text { Mixed feeding }\end{array}$ & $\begin{array}{c}\text { REF } \\
0.94(0.40-2.23) \\
0.63(0.20-1.99) \\
\end{array}$ & $\begin{array}{l}- \\
0.890 \\
0.430\end{array}$ & $\begin{array}{c}\text { REF } \\
0.92(0.40-2.12) \\
0.62(0.19-2.01) \\
\end{array}$ & $\begin{array}{c}- \\
0.850 \\
0.420 \\
\end{array}$ \\
\hline \multirow[t]{3}{*}{ Infant age, weeks } & $1.00(0.98-1.02)$ & 0.970 & $1.01(0.98-1.04)$ & 0.510 \\
\hline & \multicolumn{4}{|c|}{ Level of MMc across infancy } \\
\hline & DRR (95\% Cl) & $P$ value & Adj. DRR (95\% Cl) & $P$ value \\
\hline Maternal HIV & $0.91(0.17-4.87)$ & 0.917 & $0.70(0.26-1.92)$ & 0.494 \\
\hline $\begin{array}{l}\text { Gravidity } \\
\text { Primigravidae } \\
\text { Secondigravidae } \\
\text { Multigravidae } \\
\end{array}$ & $\begin{array}{c}\text { REF } \\
11.2(2.78-45.2) \\
0.74(0.14-3.99) \\
\end{array}$ & $\begin{array}{c}- \\
0.001 \\
0.727 \\
\end{array}$ & $\begin{array}{c}\text { REF } \\
1.95(0.43-8.94) \\
0.47(0.12-1.91) \\
\end{array}$ & $\begin{array}{l}- \\
0.388 \\
0.290\end{array}$ \\
\hline Infant sex, female & $8.79(2.40-32.0)$ & 0.001 & $4.53(1.46-14.0)$ & 0.009 \\
\hline $\begin{array}{l}\text { Feeding } \\
\text { Exclusive breastfeeding } \\
\text { Formula feeding } \\
\text { Mixed feeding }\end{array}$ & $\begin{array}{c}\text { REF } \\
0.62(0.12-3.04) \\
0.12(0.03-0.53) \\
\end{array}$ & $\begin{array}{c}- \\
0.552 \\
\mathbf{0 . 0 0 5}\end{array}$ & $\begin{array}{c}\text { REF } \\
0.24(0.05-1.13) \\
0.03(0.02-0.41) \\
\end{array}$ & $\begin{array}{c}- \\
0.072 \\
\mathbf{0 . 0 0 8}\end{array}$ \\
\hline Infant age, weeks & $1.05(1.03-1.07)$ & $<0.001$ & $1.14(1.07-1.21)$ & $<0.001$ \\
\hline $\begin{array}{l}\text { Detection of maternal mi } \\
\text { estimating equation (GE } \\
\text { equivalents }(\mathrm{gEq} \text { ) assess } \\
\text { negative binomial distribu } \\
\text { EGA: estimated gestation }\end{array}$ & $\begin{array}{l}\text { chimerism (MMc) } \\
\text { models with binon } \\
\text { d. Level of MMc ac } \\
\text { on with counts for } \\
\text { age, DRR: detec }\end{array}$ & $\begin{array}{l}\text { Ss infanc } \\
\text { distributio } \\
\text { infancy } \\
\text { microchi }\end{array}$ & $\begin{array}{l}\text { as analyzed using g } \\
\text { ontrolling for total ge } \\
\text { analyzed using GEE } \\
\text { ic and total gEq. Adj } \\
\text { : odds ratio. }\end{array}$ & $\begin{array}{l}\text { alized } \\
\text { ic } \\
\text { dels with } \\
\text { ljusted, }\end{array}$ \\
\hline
\end{tabular}


bioRxiv preprint doi: https://doi.org/10.1101/2021.03.02.432825; this version posted March 2, 2021. The copyright holder for this preprint (which was not certified by peer review) is the author/funder. All rights reserved. No reuse allowed without permission.

Table 4. Association between HIV exposure and duration of antiretroviral therapy on detection and level of maternal microchimerism at birth and across infancy.

\begin{tabular}{|c|c|c|c|c|}
\hline & \multicolumn{4}{|c|}{ Detectable MMc at birth } \\
\hline & OR (95\% Cl) & $\mathbf{P}$ value & Adj. OR (95\% Cl) & $P$ value \\
\hline \multirow[t]{3}{*}{$\begin{array}{l}\text { HIV unexposed } \\
\text { Early ART } \\
\text { Late ART } \\
\end{array}$} & $\begin{array}{c}\text { REF } \\
0.89(0.25-3.10) \\
0.73(0.19-2.74)\end{array}$ & $\begin{array}{c}- \\
0.852 \\
0.646\end{array}$ & $\begin{array}{c}\text { REF } \\
0.85(0.23-3.11) \\
0.74(0.18-2.92)\end{array}$ & $\begin{array}{c}- \\
0.802 \\
0.666\end{array}$ \\
\hline & \multicolumn{4}{|c|}{ Quantitative Level of MMc at birth } \\
\hline & $\operatorname{DRR}(95 \% \mathrm{Cl})$ & $\mathbf{P}$ value & Adj.DRR (95\% Cl) & $P$ value \\
\hline \multirow[t]{3}{*}{$\begin{array}{l}\text { HIV unexposed } \\
\text { Early ART } \\
\text { Late ART }\end{array}$} & $\begin{array}{c}\text { REF } \\
0.63(0.12-3.25) \\
0.29(0.06-0.90) \\
\end{array}$ & $\begin{array}{c}- \\
0.577 \\
\mathbf{0 . 0 3 4} \\
\end{array}$ & $\begin{array}{c}\text { REF } \\
0.50(1.55-1.64) \\
0.21(0.07-0.60) \\
\end{array}$ & $\begin{array}{c}- \\
0.254 \\
\mathbf{0 . 0 0 4} \\
\end{array}$ \\
\hline & \multicolumn{4}{|c|}{ Detectable MMc across infancy } \\
\hline & OR $(95 \% \mathrm{Cl})$ & P value & Adj.OR $(95 \% \mathrm{Cl})$ & P value \\
\hline $\begin{array}{l}\text { HIV unexposed } \\
\text { Early ARV therapy } \\
\text { Late ARV therapy }\end{array}$ & $\begin{array}{c}\text { REF } \\
1.20(0.54-2.66) \\
0.63(0.27-1.46) \\
\end{array}$ & $\begin{array}{c}- \\
0.650 \\
0.280 \\
\end{array}$ & $\begin{array}{c}\text { REF } \\
1.03(0.46-2.32) \\
0.56(0.24-1.28) \\
\end{array}$ & $\begin{array}{c}- \\
0.950 \\
0.170 \\
\end{array}$ \\
\hline & \multicolumn{4}{|c|}{ Quantitative Level of MMc across infancy } \\
\hline & DRR (95\% Cl) & P value & Adj. DRR (95\% Cl) & P value \\
\hline $\begin{array}{l}\text { HIV unexposed } \\
\text { Early ART } \\
\text { Late ART } \\
\end{array}$ & $\begin{array}{c}\text { REF } \\
1.44(0.25-8.48) \\
0.28(0.03-2.36) \\
\end{array}$ & $\begin{array}{c}- \\
0.683 \\
0.242 \\
\end{array}$ & $\begin{array}{c}\text { REF } \\
1.23(0.46-3.28) \\
0.08(0.02-0.29) \\
\end{array}$ & $\begin{array}{c}0.674 \\
<0.001 \\
\end{array}$ \\
\hline \multicolumn{5}{|c|}{$\begin{array}{l}\text { Detection of maternal microchimerism (MMc) at birth was analyzed using logistic regression } \\
\text { controlling for total genomic equivalents }(\mathrm{gEq}) \text { assessed. Quantitative levels of MMc at birth was } \\
\text { analyzed using negative binomial regression. Adjusted birth models included gravidity and infant } \\
\text { sex. Detection of MMc across infancy was analyzed using generalized estimating equation (GEE) } \\
\text { models with binomial distribution, controlling for total gEq assessed. Level of MMc across infancy } \\
\text { was analyzed using GEE models with negative binomial distribution. Adjusted infant models } \\
\text { included gravidity, infant sex, feeding status, and infant age. Adj: adjusted, ART: antiretroviral } \\
\text { therapy, EGA: estimated gestational age, OR: odds ratio, DRR: detection rate ratio. }\end{array}$} \\
\hline
\end{tabular}

